\title{
PRECISION ANALYSIS OF HEIGHT MEASUREMENTS REALIZED ON BALLASTLESS TRACK
}

The ballastless track is a relatively new track construction built in Slovak Railways. The Department of Geodesy and the Department of Railway Building and Track Management of the University of Zilina have been involved in the research project aimed to monitor such an unconventional track construction. The periodical diagnostics of the vertical and positional geometrical track parameters is realized by absolute and relative methods to determine the construction conditions under traffic loading. Precision analysis of the observation is necessary to define confidential interval of measurements errors.

Keywords: Reference network, digital levelling, Gauss-Markov model, outlier, standard deviation.

\section{Introduction}

Railway modernization in Slovakia brings apart from the social-economic and environmental advantages also technological innovations of construction layers of railway body, which will eventually contribute to the increase of the railway safety and passengers comfort. Such a technological innovation of railway body was realized in the tunnel Turecky vrch built at the railway corridor Nove Mesto nad Vahom - Puchov where the traditional railway construction was replaced by the ballastless one. The University of Zilina has been involved inthe research project aimed to monitor the behaviour of the ballastless track because it is a relatively new construction in Slovak conditions. Specifically, the Department of Railway Building and Track Management has great experience with diagnostics of the railway surface and subgrade and the Department of Geodesy monitors the geometric track position in its absolute position.

The great advantage of using geodetic methods in precise measurement of a construction is the determination of its spatial position relative to global or local reference system. Disadvantage of geodetic observations can be in the fact that precision of the measured construction depends also on the interior precision of the used reference system.

The main task of this paper is to present the geodetic measurements realized on the track ballastless construction, to demonstrate the process of precision analysis of periodical height measurements from the point of view of its conformity with the prescribed tolerances involved in the technical standards [1], [2] and [3].

\section{Observation requirements}

Present experience with using the ballastless track construction RHEDA 2000 (Fig. 1) which has been implemented into the railway body in some European countries (Germany, Spain, Netherland, etc.) shows that the great advantage of such a construction is in its positional and vertical stability, which demands less maintenance [4] and [5]. For these reasons, it is highly important to apply very precise methods to monitor the potential vertical changes of geometrical track position which can be caused by the traffic loading.

Technical norm [3] oriented on the control measurement of line constructions recommends the basic standard deviation $\bar{\sigma}_{m e t}$ with the following ratio:

$\bar{\sigma}_{m e t}=0.5 \varepsilon_{\alpha}$

where $\varepsilon_{\alpha}$ is the boundary measurement error which depends on the control parameter tolerance $\Delta x$ :

$\varepsilon_{\alpha}=0.2 \Delta x$

\footnotetext{
* ' 'Jana Izvoltova, ${ }^{1}$ Peter Pisca, ${ }^{1}$ Andrej Villim, ${ }^{2}$ Marian Mancovic

${ }^{1}$ Department of Geodesy, Faculty of Civil Engineering, University of Zilina, Slovakia

${ }^{2}$ The Institute of Foreign Languages, University of Zilina, Slovakia

E-mail: jana.izvoltova@fstav.uniza.sk
} 


\section{COMMNICOIIONS}
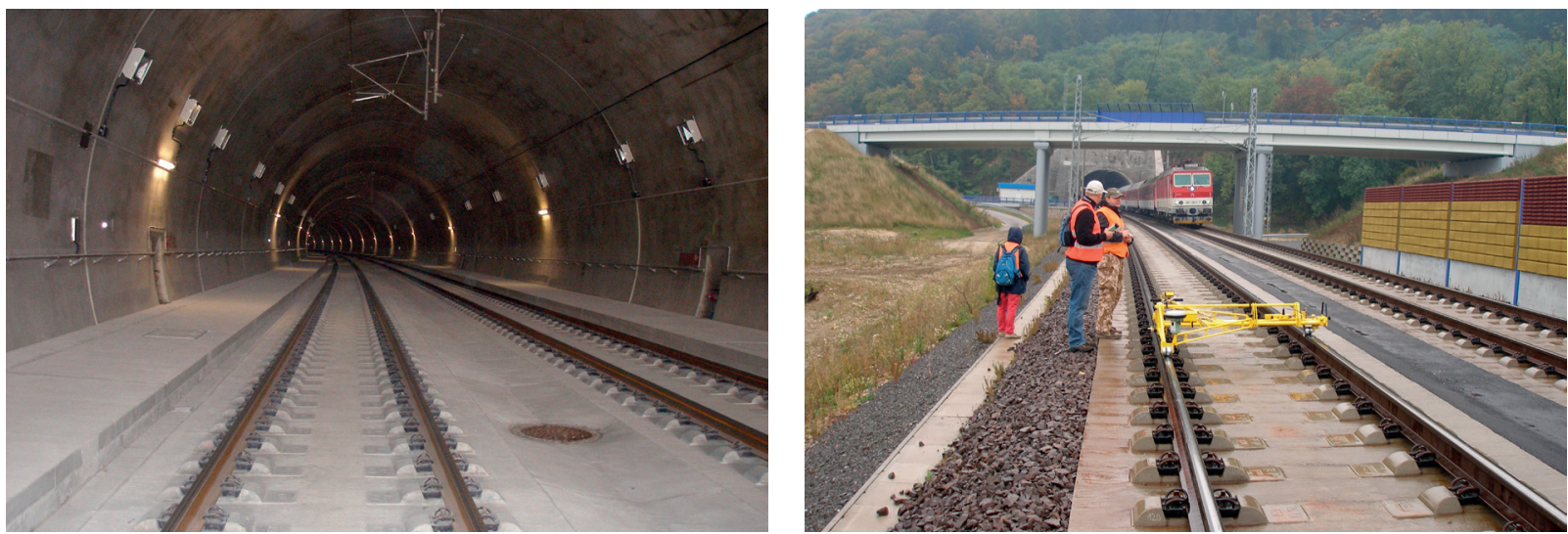

Fig. 1 Type of Ballastless Track Construction RHEDA 2000

For monitoring the ballastless track, the digital precise levelling was applied in both measurements to control the vertical stability of the reference network and track construction.

\section{Precision analysis of vertical reference network}

The precision analysis of the reference network consists both of definition of the reference network vertical stability and determination of a unit standard deviation which involves the levelling precision criteria.

The vertical stability of the reference network starts with the parameter estimation of a regression line constructed on the base of determining the elevation differences (superelevations) between all pairs of the reference points:

$\Delta_{i, i+1}^{j}=\sum_{k=i}^{n-1} \Delta h_{k, k+1}^{j}$.

The elevation differences are calculated as the levelling differences between reference points of a periodical measurement and the reference points of the first mesurement as follows:

$\Delta h_{k, k+1}^{j}=h_{k, k+1}^{j}-h_{k, k+1}^{1}$,

where the upper index $j=1,2$ represents the series of periodical measurements, lower index $i=1,2 \ldots, n-1$ means the number of calculated elevations and $n=10$ is the number of reference points (benchmarks). Providing the superelevations are normally distributed, the position of the regression line can be estimated by the least square method [6] and [7], otherwise a robust regression can be used [8] and [9]

The process of controlling the reference network stability comes to an outlier diagnostics procedure which consists in testing the extreme difference between the superelevation and the regression line. Most of the outlier detection methods belong to the statistical hypothesis testing and are based on postulate of null hypothesis:
$H_{0}: x_{e x}>x(\alpha)_{c r i t}$

where the testing value $x_{e x}$ is compared with the critical value $x(\alpha)_{\text {crit }}$ both from the same probability distribution.

For the reason of outlier detection of the vertical reference network, a robust outlier detection method was applied to test the studentized (jackknifed) residuals which are the secondary products of estimation theory applied on Gauss-Markov model. The parameter estimation primary brings a vector of unknown parameters $\hat{\beta}$, residuals $\hat{e}$ which represents the vertical displacements of superelevations from the regression line and their covariance matrices $D(\hat{\beta}), D(\hat{e})$ with their appropriate variance and covariance components [10]:

$\hat{\beta}=\left(A^{T} A\right)^{-1} A^{T} y$

$\hat{e}=y-A \hat{\beta}=\left(I-A\left(A^{T} A\right)^{-1} A^{T}\right) y$

$D(\hat{\beta})=\sigma^{2}\left(A^{T} A\right)^{-1}$

$(6 a, b, c, d)$

$D(\hat{e})=\sigma_{e}^{2}=\sigma^{2}\left(I-A\left(A^{T} A\right)^{-1} A^{T}\right)$

where $y$ represents $n \times 1$ vector of dependent observations, $A$ is an $n \times k$ design matrix and $\sigma^{2}$ is an estimation of variance calculated as follows:

$\sigma^{2}=\frac{\hat{e}^{T} \hat{e}}{n-k}$

The estimation of studentized residuals anticipates the calculation of the standardized residuals $e_{s i}$. Suppose, the residuals $\hat{e} \in N\left(0, \sigma_{e}^{2}\right)$ are normally distributed, the standardized residuals are evaluated by their transforming into standard normal distribution $e_{s i} \in N(0,1)$ :

$e_{s i}=\frac{\hat{e}_{i}-0}{\sigma_{e}}=\frac{\hat{e}}{\sigma_{e}}$,

In the conformity with the formula (6d) the particular standardized residual of $i$-th value can be evaluated from the identical equation 

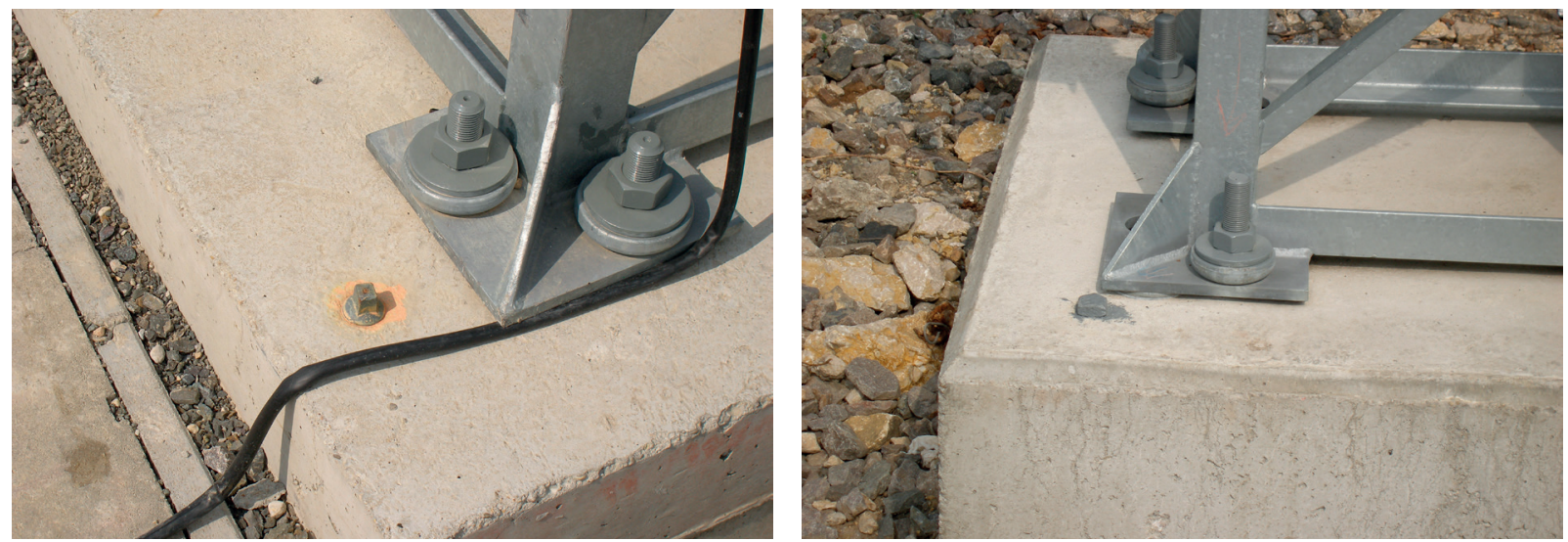

Fig. 2 Benchmarks of Vertical Reference Network

$e_{s i}=\frac{\hat{e}_{i}}{\sigma \sqrt{1-r_{i i}}}$,

where $r_{i i}$ is the diagonal element of a projection matrix:

$$
R=A\left(A^{T} A\right)^{-1} A^{T}
$$

Analogous to the idea of computation of the standardized residuals, the studentized residuals are estimated as follows:

$e_{j i}=\frac{\hat{e}_{i}}{\sigma_{-1} \sqrt{1-r_{i i}}}$,

where $\sigma_{-i}$ is the estimation of the unit standard deviation calculated without the extreme $i$-th observation. Studentized residual of $i$-th value belongs to the Student distribution with the parameter $t(\alpha)_{n-u-1}$ which represents a quantil of Student distribution with $n-u-1$ degrees of freedom and the defined probability $P=1-\alpha$. Using studentized residual as the testing value and the appropriate student parameter as a critical value, the null hypothesis can be postulated as follows:

$$
H_{0}:\left|e_{j i}\right|>t(\alpha)_{n-u-1}
$$

Reference network used for the diagnostics of vertical changes of ballastless track construction was based on ten reference points fixed bored to the concrete of the poles bottom (Fig. 2). The elevation differences (superelevations) between the benchmarks expressed in formula (3), were arranged into observed vector of the functional Gauss-Markov model which was analysed by the least square method to estimate the regression line parameters and their accuracy.

The projection matrix $R$ is calculated according to the formula (10) with the dimension $9 \times 9$ :

$R=\left(\begin{array}{rrrrrrrrr}0.3975 & 0.3206 & 0.2432 & 0.1719 & 0.1030 & 0.0391 & -0.0189 & -0.0920 & -0.1644 \\ & 0.2644 & 0.2077 & 0.1556 & 0.1052 & 0.0584 & 0.0160 & -0.0375 & -0.0905 \\ & & 0.1720 & 0.1392 & 0.1074 & 0.0779 & 0.0511 & 0.0175 & -0.0159 \\ & & & 0.1240 & 0.1094 & 0.0958 & 0.0835 & 0.0680 & 0.0526 \\ & & & & 0.1113 & 0.1131 & 0.1148 & 0.1168 & 0.1189 \\ & & & & & 0.1292 & 0.1438 & 0.1622 & 0.1804 \\ & & & & & & 0.1702 & 0.2033 & 0.2362 \\ & & & & & & & 0.2551 & 0.3065 \\ & & & & & & & & 0.3762\end{array}\right)$, 
Diagonal components of the projection matrix $r_{i i}$ are needed to evaluate vector of residuals $\hat{e}$, standardized residuals $\hat{e}_{s}$ and the studentized residuals $\hat{e}_{j}$ :

$\hat{e}=\left(\begin{array}{r}0.1828 \\ 0.1540 \\ -0.1740 \\ 0.3393 \\ -0.6183 \\ -0.4903 \\ 0.1317 \\ 0.3499 \\ 0.1249\end{array}\right), \hat{e}_{s}=\left(\begin{array}{r}0.6292 \\ 0.4797 \\ -0.5108 \\ 0.9685 \\ -1.7523 \\ -1.4040 \\ 0.3862 \\ 1.0833 \\ 0.4226\end{array}\right), \hat{e}_{j}=\left(\begin{array}{r}0.8019 \\ 0.6109 \\ -0.6505 \\ 1.2334 \\ -2.2317 \\ -1.7880 \\ 0.4919 \\ 1.3796 \\ 0.5382\end{array}\right)$.

$(14 a, b, c)$

The value of standard deviation $\sigma$ estimated from the full residual vector and unit of standard deviation $\sigma_{-5}$ estimated without the extreme value of residuals are following:

$$
\begin{aligned}
& \sigma=0.37 \mathrm{~mm} \\
& \sigma_{-5}=0.29 \mathrm{~mm}
\end{aligned}
$$

The estimated vector of residuals (14a) was indicative of the fifth extreme value $e_{5}=-0.6183$ and so the appropriate value of studentized residual $e_{j 5}=-2.2317$ had to be exposed to null hypothesis as follows:

$$
H_{0}:|-2.2317|>t(\alpha)_{n-u-1}
$$

Suppose that the model redundancy is $n-u-1$ and the Student coefficient is $t(0.05)_{n-u-1}=1.9432$, the fifth superelevation in the reference network is considered to be outlier with probability $P=95 \%$. Owing to the fact that null hypothesis is confirmed, measurements and evaluations proceed without this extreme superelevation.

\section{Precision analysis of track measurement}

The main output of the precision analysis of vertical reference network consists in defining its vertical stability which is important for additional monitoring of the track construction and in estimation of levelling precision represented by the value $\sigma_{h}$.

The precision of height differences of the measured track construction is estimated according to the equation:

$\sigma_{h}=\sigma_{0} \sqrt{L}=0.41 \mathrm{~mm}$

The vertical displacement of an observed point is evident if it exceeds the tolerance boundaries which are determined as a double value of standard deviation $\sigma_{h}$ :

$\bar{x}-2 \sigma_{h} \leq \Delta \leq \bar{x}+2 \sigma_{h}$

The observed results and the analysis of precision are graphically demonstrated in Fig. 3. The measured vertical

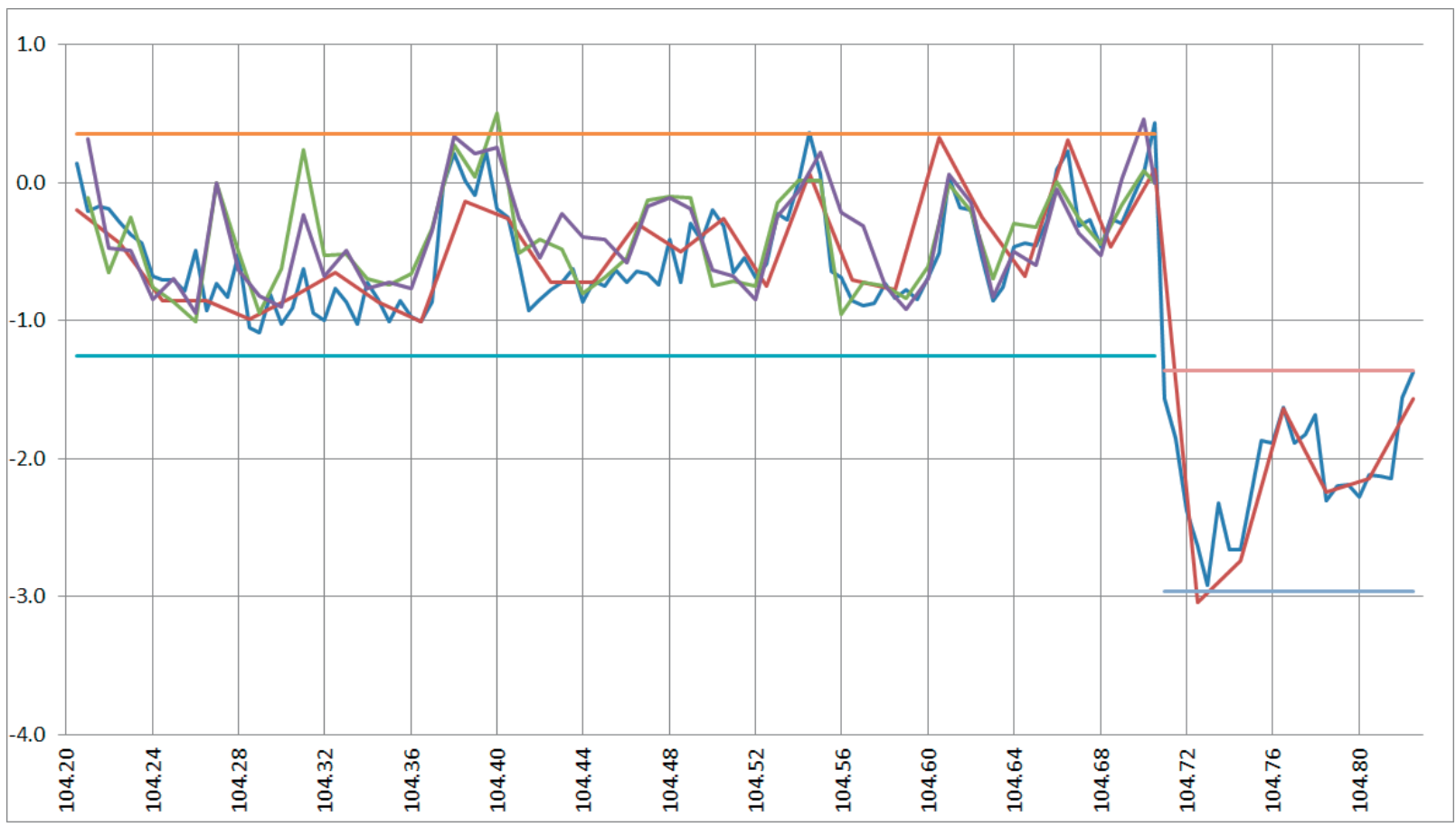

Fig. 3 Analysis of vertical stability of track 1 
changes of the ballastless track construction did not exceed the confidential interval and so the vertical displacements of the track construction are not confirmed. Another situation occurs in the railway part in $\mathrm{km} 104.705-104.820$, built on the traditional track construction where the track construction decreased by more than two millimetres (see Fig. 3).

\section{Contributions}

Precision analysis of height measurements realized on the ballastless track consists both of analysis of vertical reference network and precision analysis of the track measurements. The first one brought the view on stability of the reference system and standard deviation as the main characteristic of the used levelling method (15). Precision analysis of track measurements consists of defining the standard deviation of height differences (17) and the confidential interval (18) which qualifies the evident vertical displacements of track construction.

The precision analysis of geodetic observations realized on the ballastless track construction was performed in the conformity with the appropriate technical standards. Precision of used digital levelling satisfies the demands defined for observation of such an unconventional track construction. Since the vertical stability of the ballastless track construction has been confirmed by both measurements, the vertical changes of traditional track construction have been registered.

\section{Acknowledgement}

This article is the result of the implementation of the project: "Innovation and internationalization of education - the means to increase the quality of the University of Zilina in the European educational space (ITMS: 26110230079) supported by the Research \& Development Operational Programme funded by the ERDF.

This article is the result of the implementation of the project VEGA No. 1/0597/14 "Analysis of methods used to measure the unconventional railway track construction from the point of view of accuracy and reliability" supported by the Scientific Grant Agency of the Ministry of Education, science, research and sport of the Slovak Republic and the Slovak Academy of Sciences.

\section{References}

[1] STN 730202 Precision of Geometric Parameters in the Construction (in Slovak). General requirement, Bratislava 1985

[2] STN 73 0212-6 Precision of Geometric Parameters in the Construction (in Slovak). Precision control, Bratislava 1993

[3] STN 730275 Precision of Geometric Parameters in the Construction (in Slovak). Control measurement of line structures, Bratislava 1985

[4] RHEDA 2000® Ballast-less Track System. http://www.railone.com/en/main-nav/products/railways-and-commuter-traffic/ballastlesstrack-systems.html

[5] IZVOLT, L., HODAS, S.: Modernisation of Railway Infrastructure in the Slovak Republic. COMPRAIL XIII. intern. Conference on Design and Operation in Railway Engineering, WIT Press Southampton : Boston, ISBN 978-1-84564-616-5

[6] MARCAK, P., KUBACEK. L.: The Problem of the System of Height Reference in Determining the Settling of Foundations and Buildings. Studia Geophysica et Geodaetica, 18, 33-46, 1974

[7] SIMA, J., KOTKA, V., PISCA, P., SEIDLOVA, A.: Geodetic Work for Reconstruction and Building of Narrow-gauge Railway. XIII. intern. Slovak-Poland-Russian geodetic days : Liptovsky Jan, 84-88, 2007, ISBN 978-80-969692-0-3

[8] CEBECAUER, D.: Reference System used for Measurement of Vertical Shifts in Line Buildings (in Slovak). Geodeticky a kartograficky obzor, vol. 12, No. 36/78, 300-303, 1990

[9] ROUSSEEUW, P. J., LEROY, A. M.: Robust Regression and Outlier Detection, Wiley \& Sons : New Jersey 2003, 329 p., ISBN 0-47148855-0

[10] MUZIK, J., KOVARIK, K., SITANYOVA, D.: Meshless Analysis of an Embankment Using Local Galerkin Radial Point Interpolation Method (LGRPIM). Communications - Scientific Letters of the University of Zilina, No. 2, 34-40, 2013, ISSN $1335-4205$. 